\title{
Effective potential for a covariantly constant gauge field in curved spacetime
}

\author{
Emilio Elizalde ${ }^{a, b, *}$, Sergei D. Odintsov ${ }^{b, \dagger}$, and August Romeo ${ }^{a, \ddagger}$ \\ ${ }^{a}$ Centre for Advanced Studies CEAB, CSIC, Camí de Santa Bàrbara, 17300 Blanes \\ ${ }^{b}$ Dept ECM and IFAE, Fac. Physics, University of Barcelona, Diagonal 647, 08028 Barcelona
}

\begin{abstract}
We discuss the influence of gravitational effects on the stabilization of the chromomagnetic vacuum. The one-loop effective potential for a covariantly constant $\mathrm{SU}(2)$ gauge field in $\mathbf{S}^{2} \times \mathbf{R}^{2}$ and $\mathbf{T}^{2} \times \mathbf{R}^{2}$ is calculated. A possibility of curvature-induced phase transitions between zero and nonzero chromomagnetic vacua is found - what is also confirmed through the calculation of the renormalization group (RG) improved effective potential on constant-curvature spaces with small curvature. Numerical evaluation indicates that for some curvatures the imaginary part of the effective potential disappears (gravitational stabilization of the chromomagnetic vacuum occurs).
\end{abstract}

\section{Introduction}

The study of the effective Lagrangian for covariantly constant gauge fields in nonabelian gauge theories showed the possibility of existence for a nonzero minimum (the so-called chromomagnetic vacuum [1]). Unfortunately, such a state could not be the true ground state of the theory due to the presence of unstable field modes which lead to the appearance of an imaginary part in the effective Lagrangian [2] (see also [3]-[6] and references therein). Several proposals for stabilizing the vacuum may be considered. For example, in the interesting paper [5] it has been shown that a stable ground state in electroweak theory may be achieved for a space-dependent magnetic field.

In the present letter, we investigate the effects of nonzero curvature on the stabilization of the chromomagnetic vacuum for a pure gauge theory in curved spacetime. Using zeta-regularization methods we calculate the effective potential for an $\mathrm{SU}(2)$ covariantly constant gauge field in a spacetime of the form $\mathbf{M}^{2} \times \mathbf{R}^{2}$, where $\mathbf{M}^{2}$ is a curved manifold - here the case of the twodimensional sphere and two-dimensional torus will be considered- and $\mathbf{R}^{2}$ a flat plane. The nonzero components of the background gauge field are them embedded into $\mathbf{R}^{2}$. An analysis of the phase structure from the effective potential shows the chance of curvature-induced phase transitions between zero and nonzero chromomagnetic ground states. Moreover, our study indicates the possibility of removing the imaginary part by curvature effects, thus stabilizing the

${ }^{*}$ E-mail: eli@zeta.ecm.ub.es

†On leave of absence from Tomsk Pedagogical University, 634041 Tomsk, Russia. E-mail: odintsov@ecm.ub.es

${ }_{\ddagger}^{\ddagger}$ E-mail: august@ceab.es 
chromomagnetic vacuum. The possibility of curvature-induced phase transitions is also verified in sect. 3 through the calculation of the RG-improved effective potential in curved spacetime for small curvatures (where quasi-local expansions can be used).

\section{Gauge field effective potential on an $\mathrm{S}^{2} \times \mathrm{R}^{2}$ background}

Let us consider a pure $\mathrm{SU}(N)$ gauge theory on an $\mathbf{S}^{2} \times \mathbf{R}^{2}$ spacetime background with a nonzero covariantly constant background gauge field. Like in flat spacetime, the calculation of the one-loop effective action is quite straightforward, giving the result

$$
\Gamma^{(1)}[\bar{A}]=\frac{1}{2} \operatorname{Tr} \ln \left[\bar{\Theta}_{\mu \nu}^{a b}\right]-\operatorname{Tr} \ln \left[\left(-\bar{D}^{2}\right)^{a b}\right]
$$

where the operators present are

$$
\begin{aligned}
& \bar{\Theta}_{\mu \nu}^{a b}=-g_{\mu \nu}\left(\bar{D}_{\lambda} \bar{D}^{\lambda}\right)^{a b}+2 i g\left(T^{c}\right)^{a b} \bar{F}_{\mu \nu}^{c}+(1-1 / \xi)\left(\bar{D}_{\mu} \bar{D}_{\nu}\right)^{a b}-\delta^{a b} R_{\mu \nu}, \quad \xi=1 \\
& \bar{D}_{\mu}^{a b}=\delta^{a b} \nabla_{\mu}-i g\left(T^{c}\right)^{a b} \bar{A}_{\mu}^{c}
\end{aligned}
$$

The $T^{a}$ matrices are the colour $\mathrm{SU}(N)$ generators, which we will suppose written in the adjoint representation. Colour $\mathrm{SU}(N)$ indices are inset in such a way that

$$
\begin{aligned}
& \bar{A}_{\mu}=n^{a} \bar{A}_{\mu}^{a}, \\
& \bar{F}_{\mu \nu}=n^{a} \bar{F}_{\mu \nu}^{a},
\end{aligned}
$$

being $n$ a unit-norm vector along some given colour space direction. Then

$$
\bar{\Theta}_{\mu \nu}^{a b}=-g_{\mu \nu}\left[\left(\nabla_{\lambda}-i g\left(n^{c} T^{c}\right) \bar{A}_{\lambda}\right)^{2}\right]^{a b}+i g\left(n^{c} T^{c}\right)^{a b} \bar{F}_{\mu \nu}-\delta^{a b} R_{\mu \nu} .
$$

The Tr ln calculations in (11) will be performed by the zeta function method. In view of that equation, the function whose derivative at $s=0$ will provide the effective action is the linear combination

$$
-\frac{1}{2} \zeta_{\bar{\Theta} / \mu^{2}}(s)+\zeta_{-\bar{D}^{2} / \mu^{2}}(s),
$$

where the subscripts mean the operators to which each zeta function is associated (for an introduction to zeta-regularization in full detail, see the recent monographies [7]). Renormalization of gauge theories in curved spacetime has been thoroughly discussed in refs. [8] (for a review and introduction to quantum field theory in curved spacetime, see [9]).

We start by diagonalizing the colour-structure. Formally, one identifies the eigenvalues of the colour matrices:

$$
\left(n^{c} T^{c}\right) \text { with eigenvalues } \nu^{a}, a=1, \ldots, N^{2}-1
$$

Thus, we find the colour 'eigenoperators'

$$
\bar{\Theta}_{\mu \nu}^{a}=-g_{\mu \nu}\left(\nabla_{\lambda}-i g \nu^{a} \bar{A}_{\lambda}\right)^{2}+i g \nu^{a} \bar{F}_{\mu \nu}-R_{\mu \nu}, a=1, \ldots, N^{2}-1
$$

Now, the selected type of background comes into play. We choose, as a background gauge field,

$$
\bar{A}_{\mu}=-\frac{1}{2} \bar{F}_{\mu \nu} x^{\nu}
$$


with constant $\bar{F}_{\mu \nu}$. It is covariantly constant, i.e. it satisfies $\nabla_{\mu} \bar{A}^{\mu}=0$.

The spacetime in which we shall work is $\mathbf{S}^{\mathbf{2}} \times \mathbf{R}^{2}$. Ordering the coordinates in the way $\left(\theta, \varphi, x_{1}, x_{2}\right)$, we consider a purely colour-magnetic case for which

$$
\left(\bar{F}_{\mu \nu}\right)=\left(\begin{array}{cccc}
0 & 0 & 0 & 0 \\
0 & 0 & 0 & 0 \\
0 & 0 & 0 & H \\
0 & 0 & -H & 0
\end{array}\right)
$$

As a result,

$$
\Delta^{a} \equiv\left(\nabla_{\lambda}-i g \nu^{a} \bar{A}_{\lambda}\right)^{2}=\nabla^{2}+i g \nu^{a} H\left(x_{1} \partial_{2}-x_{2} \partial_{1}\right)-\frac{g^{2}\left(\nu^{a}\right)^{2}}{4} H^{2}\left(x_{1}^{2}+x_{2}^{2}\right) .
$$

Since our spacetime is $\mathbf{S}^{\mathbf{2}} \times \mathbf{R}^{2}, \nabla^{2}=\nabla_{\mathbf{S}^{2}}^{2}+\partial_{1}^{2}+\partial_{2}^{2}$. In terms of the new variables

$$
\left\{\begin{aligned}
Q_{1} & \equiv i \partial_{1}+\frac{g \nu^{a}}{2} H x_{2} \\
Q_{2} & \equiv i \partial_{2}-\frac{g \nu^{a}}{2} H x_{1}
\end{aligned}\right.
$$

we can write

$$
\Delta^{a}=\nabla_{\mathbf{S}^{2}}^{2}-Q_{1}^{2}-Q_{2}^{2}
$$

What is more, given that $\left[Q_{1}, Q_{2}\right]=-i g \nu^{a} H$, one may take the representation

$$
Q_{2}=i g \nu^{a} H \frac{\partial}{\partial Q_{1}}
$$

and put

$$
\begin{aligned}
\Delta^{a} & =\nabla_{\mathbf{S}^{2}}^{2}-Q_{1}^{2}+\left(g \nu^{a} H\right)^{2} \frac{\partial^{2}}{\partial Q_{1}^{2}} \\
& =\nabla_{\mathbf{S}^{2}}^{2}-2 \mathcal{H}_{\omega^{a}}
\end{aligned}
$$

where $\mathcal{H}_{\omega^{a}}$ indicates the QM-Hamiltonian of a harmonic oscillator with frequency

$$
\omega^{a} \equiv g H\left|\nu^{a}\right|
$$

Let us now diagonalize the spacetime-index structure of

$$
\bar{\Theta}_{\mu \nu}^{a}=-g_{\mu \nu} \Delta^{a}+i g \nu^{a} \bar{F}_{\mu \nu}-R_{\mu \nu} .
$$

Taking into account that

$$
R_{\mu \nu}=\left\{\begin{array}{l}
g_{\mu \nu}=\operatorname{diag}\left(g_{\theta \theta}, g_{\varphi \varphi}, 1,1\right) \\
\frac{1}{\rho^{2}} g_{\mu \nu}, \\
0, \quad \text { when } \mu, \nu \text { are } \mathbf{S}^{2} \text { indices }
\end{array}\right.
$$

( $\rho$ is the radius of $\mathbf{S}^{2}$ ) and the particular choice of $\bar{F}_{\mu \nu}$, we write $\bar{\Theta}_{\mu \nu}^{a}$ in spacetime-matrix form:

$$
\left(\bar{\Theta}_{\mu \nu}^{a}\right)=\left(\begin{array}{cccc}
-g_{\theta \theta}\left(\Delta^{a}+1 / \rho^{2}\right) & 0 & 0 & 0 \\
0 & -g_{\varphi \varphi}\left(\Delta^{a}+1 / \rho^{2}\right) & 0 & 0 \\
0 & 0 & -\Delta^{a} & 2 i g \nu^{a} H \\
0 & 0 & -2 i g \nu^{a} H & -\Delta^{a}
\end{array}\right)
$$


Its diagonalization produces again four 'eigenoperators', which are

$$
-g_{\theta \theta}\left(\Delta^{a}+1 / \rho^{2}\right), \quad-g_{\varphi \varphi}\left(\Delta^{a}+1 / \rho^{2}\right), \quad-\Delta^{a} \pm 2 g\left|\nu^{a}\right| H
$$

All of them can be recast into the common notation

$$
\bar{\Theta}_{\mu}^{a} \equiv-g_{\mu \mu}\left(\Delta^{a}+m_{\mu}^{a}\right),\left\{\begin{array}{c}
m_{\theta}^{a}=m_{\varphi}^{a}=1 / \rho^{2} \\
m_{1}^{a}=-m_{2}^{a}=-2 \omega^{a},
\end{array}\right.
$$

(where (15) has been recalled) and one just has to consider the eigenvalues of $-\left(\Delta^{a}+m_{\mu}^{a}\right)$, which are

$$
\frac{1}{\rho^{2}} l(l+1)+2 \omega^{a}\left(n+\frac{1}{2}\right)-m_{\mu}^{a} \equiv \Lambda_{\mu}^{a}(n, l), \text { with degeneracy } d_{l}=2 l+1, l \in \mathbf{N}, n \in \mathbf{N}
$$

Although not calling it this name, zeta-function regularization was applied to problems of similar nature as early as the time of ref. [10] (for a review, see [7]). Now that the spectrum is known, we construct the zeta function for the whole $\bar{\Theta}$ operator

$$
\zeta_{\bar{\Theta}}(s)=\operatorname{Tr} \bar{\Theta}^{-s}=\sum_{a} \sum_{\mu} \int d^{4} x \sqrt{g} \operatorname{tr}\left\langle x\left|\left(\bar{\Theta}_{\mu}^{a}\right)^{-s}\right| x\right\rangle
$$

where tr runs over the harmonic oscillator and angular momentum quantum numbers (so that $\operatorname{Tr}$ runs over everything). However, the above eigenvalues were referred to the $Q_{1}$ - and not the $x$ - coordinates. Introducing the adequate spectral resolution of the identity and bearing in mind the property $\int d Q_{1}\left|\left\langle x \mid Q_{1}, n, l\right\rangle\right|^{2}=\frac{\omega^{a}}{2 \pi}$, we find

$$
\zeta_{\bar{\Theta}}(s)=\frac{\Omega}{2 \pi\left(4 \pi \rho^{2}\right)} \sum_{a} \omega^{a} \sum_{\mu} \sum_{n} \sum_{l} d_{l}\left[\Lambda_{\mu}^{a}(n, l)\right]^{-s},
$$

where $\Omega \equiv \int \sqrt{g} d^{4} x=4 \pi \rho^{2} \int d x_{1} d x_{2}$ denotes the total spacetime volume. Using the form of $\Lambda_{\mu}^{a}(n, l)$ given by (21) and the dimensionless variables

$$
\widehat{\omega}^{a} \equiv \rho^{2} \omega^{a}, \quad \widehat{m}_{\mu}^{a} \equiv \rho^{2} m_{\mu}^{a},
$$

this zeta function is more conveniently expressed as

$$
\zeta_{\bar{\Theta}}(s)=\frac{\Omega}{8 \pi^{2} \rho^{2}} \rho^{2 s} \sum_{a} \omega^{a} \sum_{\mu} \zeta\left(s ; \widehat{\omega}^{a}, \widehat{m}_{\mu}^{a}\right),
$$

where

$$
\zeta(s ; \widehat{\omega}, \widehat{m}) \equiv \sum_{l=0}^{\infty} \sum_{n=0}^{\infty}(2 l+1)\left[l(l+1)+2 \widehat{\omega}\left(n+\frac{1}{2}\right)-\widehat{m}\right]^{-s} .
$$

Doing a Mellin transform, summing the $n$-series afterwards, and making a trivial variable change (26), can be put into the integral form

$$
\zeta(s ; \widehat{\omega}, \widehat{m})=\frac{1}{(2 \widehat{\omega})^{s}} \frac{1}{\Gamma(s)} \int_{0}^{\infty} d u u^{s-1} \frac{e^{-\frac{u}{2}\left(1-\frac{\widehat{m}}{\widehat{\omega}}\right)}}{1-e^{-u}} Y_{\mathrm{S}^{2}}\left(\frac{u}{2 \widehat{\omega}}\right) .
$$


The advantage here is that the quantity

$$
Y_{\mathrm{S}^{2}}(t) \equiv \sum_{l=0}^{\infty}(2 l+1) e^{-t l(l+1)}
$$

is in fact the integrated heat-kernel of the (-Laplacian) operator on an $\mathbf{S}^{2}$ sphere of unit radius ( $t$ has absorbed the radius dependence, as this variable stands for $\frac{u}{2 \widehat{\omega}}=\frac{u}{2 \rho^{2} \omega}$ ).

The $-\bar{D}^{2}$ operator is similar but for all the $m_{\mu}^{a}$ pieces additional to $\Delta^{a}$ which are now absent. Since the spacetime structure is already diagonal, we do not have to take the final $\sum_{\mu}$ for these eigenvalues, and one can straightforwardly put

$$
\zeta_{-\bar{D}^{2}}(s)=\frac{\Omega}{8 \pi^{2} \rho^{2}} \rho^{2 s} \sum_{a} \omega^{a} \zeta\left(s ; \widehat{\omega}^{a}, 0\right) .
$$

Next we go on to the dimensionless versions of both operators by introducing the arbitrary mass scale $\mu$, and form the linear combination (5):

$$
-\frac{1}{2} \zeta_{\bar{\Theta} / \mu^{2}}(s)+\zeta_{-\bar{D}^{2} / \mu^{2}}(s)=\frac{\Omega}{8 \pi^{2} \rho^{2}}(\rho \mu)^{2 s} \sum_{a} \omega^{a}\left[-\frac{1}{2} \sum_{\lambda} \zeta\left(s ; \widehat{\omega}^{a}, \widehat{m}_{\lambda}^{a}\right)+\zeta\left(s ; \widehat{\omega}^{a}, 0\right)\right] .
$$

As already observed, the derivative of this function at $s=0$ yields the effective action. Further, the representation (27) enables us to write

$$
\begin{gathered}
-\frac{1}{2} \sum_{\lambda} \zeta\left(s ; \widehat{\omega}^{a}, \widehat{m}_{\lambda}^{a}\right)+\zeta\left(s ; \widehat{\omega}^{a}, 0\right)= \\
\frac{1}{\left(2 \widehat{\omega}^{a}\right)^{s}} \frac{1}{\Gamma(s)} \int_{0}^{\infty} d u u^{s-1} \frac{e^{-\frac{u}{2}}}{1-e^{-u}}\left(-\frac{1}{2} \sum_{\lambda=\theta, \varphi, 1,2} e^{\frac{u}{2} \frac{\widehat{m}_{\lambda}^{a}}{\widehat{\omega}^{a}}}+1\right) Y_{\mathrm{S}^{2}}\left(\frac{u}{2 \widehat{\omega}^{a}}\right) .
\end{gathered}
$$

Recalling (20) one realizes that $\sum_{\lambda=\theta, \varphi, 1,2} e^{\frac{u}{2} \frac{\widehat{m}_{\lambda}^{a}}{\widehat{\omega}^{a}}}=2 e^{\frac{u}{2 \widehat{\omega}^{a}}}+2 \cosh u$. Therefore, (30) reads

$$
\begin{gathered}
-\frac{1}{2} \zeta_{\bar{\Theta} / \mu^{2}}(s)+\zeta_{-\bar{D}^{2} / \mu^{2}}(s)= \\
\frac{\Omega}{8 \pi^{2}} \sum_{a} \frac{\left(\omega^{a}\right)^{2}}{\widehat{\omega}^{a}}\left(\frac{\mu^{2}}{2 \omega^{a}}\right)^{s} \frac{1}{\Gamma(s)} \int_{0}^{\infty} d u u^{s-1} \frac{e^{-\frac{u}{2}}}{1-e^{-u}}\left(-e^{\frac{u}{2 \widehat{\omega}^{a}}}-\cosh u+1\right) Y_{S^{2}}\left(\frac{u}{2 \widehat{\omega}^{a}}\right) .
\end{gathered}
$$

\subsection{Large- $\rho$ expansion}

As is well known, the small-t asymptotic expansion for the heat-kernel of a Laplacian-like operator on a two-dimensional manifold $\mathcal{M}$ without boundaries has the form

$$
Y_{\mathcal{M}}(t) \sim \frac{1}{4 \pi t}\left[A_{0}+A_{1} t+O\left(t^{2}\right)\right] \equiv \frac{1}{t}\left[a_{0}+a_{1} t+O\left(t^{2}\right)\right] .
$$

In particular, for the Laplacian on $\mathcal{M}=S^{2}$ the heat-kernel $Y_{\mathrm{S}^{2}}$ (28) gives rise to the specific values 111

$$
a_{0}=1, a_{1}=-\frac{1}{3}, \ldots
$$


Since $\widehat{\omega}^{a}=\rho^{2} \omega^{a}=\rho^{2} g H\left|\nu^{a}\right|$, expansions in $1 / \widehat{\omega}$ are actually expansions in $1 / \rho^{2}$. Thus, calling $\frac{u}{2 \widehat{\omega}^{a}} \equiv t$, one finds

$$
\left(-e^{t}-\cosh u+1\right) Y_{\mathrm{S}^{2}}(t) \sim-\left[a_{0} \frac{1}{t} \cosh u+a_{0}+a_{1} \cosh u+O(t)\right]
$$

Putting this series into (32), we integrate term by term with the help of the Hurwitz zeta function integral representation

$$
\zeta_{H}(z, \alpha)=\frac{1}{\Gamma(z)} \int_{0}^{\infty} d u u^{z-1} \frac{e^{-\alpha u}}{1-e^{-u}}, \operatorname{Re} z>1 .
$$

Substituting afterwards the result into (32) one gets

$$
\begin{gathered}
-\frac{1}{2} \zeta_{\bar{\Theta} / \mu^{2}}(s)+\zeta_{-\bar{D}^{2} / \mu^{2}}(s)= \\
-\frac{\Omega}{8 \pi^{2}} \sum_{a} 2\left(\omega^{a}\right)^{2}\left(\frac{\mu^{2}}{2 \omega^{a}}\right)^{s}\left\{\frac{a_{0}}{2} \frac{1}{s-1}\left[\zeta_{H}\left(s-1, \frac{3}{2}\right)+\zeta_{H}\left(s-1,-\frac{1}{2}\right)\right]\right. \\
\left.+\left[a_{0} \zeta_{H}\left(s, \frac{1}{2}\right)+\frac{a_{1}}{2}\left(\zeta_{H}\left(s, \frac{3}{2}\right)+\zeta_{H}\left(s,-\frac{1}{2}\right)\right)\right] \frac{1}{2 \widehat{\omega}^{a}}+O\left(\frac{1}{\left(2 \widehat{\omega}^{a}\right)^{2}}\right)\right\}
\end{gathered}
$$

It will now be easy to take the derivative at $s=0$. When doing so, we shall recall the identities

$$
\begin{aligned}
\zeta_{H}(0, x) & =-B_{1}(x)=\frac{1}{2}-x, \\
\zeta_{H}^{\prime}(0, x) & =-\frac{1}{2} \ln (2 \pi)+\ln \Gamma(x), \\
\zeta_{H}(-1, x) & =-\frac{1}{2} B_{2}(x)=-\frac{1}{2}\left(x^{2}-x+1 / 6\right),
\end{aligned}
$$

and observe that imaginary parts emerge from

$$
\left\{\begin{aligned}
\zeta_{H}^{\prime}\left(s,-\frac{1}{2}\right) & =\zeta_{H}^{\prime}\left(s, \frac{1}{2}\right)-\left(-\frac{1}{2}\right)^{-s} \ln \left(-\frac{1}{2}\right), \\
\ln \left(-\frac{1}{2}\right) & =\ln \left(\frac{1}{2}\right)-i \pi
\end{aligned}\right.
$$

where the complex argument determination taken is that from refs. [3]. Thus

$$
\begin{gathered}
\Gamma^{(1)}=-\frac{1}{2} \zeta_{\bar{\Theta} / \mu^{2}}^{\prime}(0)+\zeta_{-\bar{D}^{2} / \mu^{2}}^{\prime}(0)= \\
-\frac{\Omega}{8 \pi^{2}} \sum_{a=1}^{N^{2}-1} 2\left(\omega^{a}\right)^{2} \quad \\
\left\{\frac{a_{0}}{2} \frac{11}{12} \ln \left(\frac{\mu^{2}}{2 \omega^{a}}\right)+\frac{a_{0}}{2}\left[\frac{11}{12}-\zeta_{H}^{\prime}\left(-1, \frac{3}{2}\right)-\zeta_{H}^{\prime}\left(-1, \frac{1}{2}\right)+\frac{1}{2} \ln \left(\frac{1}{2}\right)+i \frac{\pi}{2}\right]\right. \\
\left.+\frac{1}{2 \widehat{\omega}^{a}}\left[-\frac{\left(a_{0}+a_{1}\right)}{2} \ln 2+i \frac{a_{1}}{2} \pi\right]+O\left(\frac{1}{\left(2 \widehat{\omega}^{a}\right)^{2}}\right)\right\} .
\end{gathered}
$$

While the leading part for $\rho \rightarrow \infty$ amounts to the result in [3] — which is a generalization of Savvidy's one [1] - the next-to-leading contribution provides the first curvature correction and is, in fact, linear in the curvature. Let us now consider the particular case of $N=2$ in this approximation. For $\mathrm{SU}(2)$, the adjoint representation yields $\left\{\left|\nu^{a}\right|, a=1, \ldots, N^{2}-1\right\}=\{1,1,0\}$. As a result of this, and recalling (15), (24),

$$
\frac{\Gamma^{(1)}}{\Omega}=a_{0}(g H)^{2}\left[\frac{11}{48 \pi^{2}}\left(\ln \frac{g H}{\mu^{\prime 2}}-\frac{1}{2}\right)-i \frac{1}{8 \pi}\right]+\frac{1}{4 \pi^{2}} \frac{g H}{\rho^{2}}\left[-\frac{\left(a_{0}+a_{1}\right)}{2} \ln 2+i \frac{a_{1}}{2} \pi\right],
$$


where the new arbitrary scale $\mu^{\prime}$ is related to the initial arbitrary scale $\mu$ by the transformation

$$
\ln \mu^{\prime 2}=\ln \mu^{2}-\frac{5}{11} \ln 2+\frac{1}{2}-\frac{11}{12}\left[-\zeta_{H}^{\prime}\left(-1, \frac{3}{2}\right)-\zeta_{H}^{\prime}\left(-1, \frac{1}{2}\right)\right] .
$$

¿From here on we will rename $\mu^{\prime}$ into $\mu$. Next, we turn to the 1-loop effective potential $V$ including the classical contribution, namely

$$
V=\frac{H^{2}}{2}+\frac{\Gamma^{(1)}}{\Omega}
$$

whose real and imaginary parts read, after setting the values (34), are given by

$$
\begin{aligned}
& \operatorname{Re} V(H, \rho)=\frac{H^{2}}{2}+\frac{11}{48 \pi^{2}}(g H)^{2}\left(\ln \frac{g H}{\mu^{2}}-\frac{1}{2}\right)+\frac{1}{4 \pi^{2}} \frac{g H}{\rho^{2}} \frac{1}{3} \ln 2 \\
& \operatorname{Im} V(H, \rho)=\frac{1}{8 \pi}\left[-(g H)^{2}+\frac{1}{3} \frac{g H}{\rho^{2}}\right] .
\end{aligned}
$$

They are plotted in Fig. 1 in solid and dashed line, respectively, for several values of $\rho$, including the flat space case corresponding to $\rho \rightarrow \infty$, in the order -from bottom up: flat space, $\rho=$ $10,3,2.21,2.03,1.5,1$. For this plot we have set $g=0.1$ and the arbitrary $\mu^{2}$ scale has been chosen to be $\mu^{2}=g e^{24 \pi^{2} /\left(11 g^{2}\right)}$ so that the flat space minimum of Re $V$ occurs at $H=1$. Thus, we can see how the Re $V$ minima are shifted to lower $H$-values as $\rho$ decreases. Moreover, a curvatureinduced symmetry-breaking phase transition takes place at $\rho_{c} \simeq 2.03$ : for $\rho$ larger than this value, the global minimum is located at some $H_{\min }>0$; otherwise $H_{\min }=0$. We also observe that the zero of the imaginary part and the minimum of the real part are in general different, but at $\rho_{s} \simeq 2.21$ they coincide. This indicates a possible stabilization of the magnetic vacuum due to gravitation. In the fourth section we shall calculate RG improved effective potential and find also the possibility of curvature-induced phase transitions.

\section{Gauge field effective potential on a $T^{2} \times R^{2}$ background}

Let the radii of the torus be $L_{1}$ and $L_{2}$. For the rest we shall use the same notation as in sect. 2, but this time the curvature will be taken to be zero. As a result, for the eigenvalues of $-\left(\Delta^{a}+m_{\mu}^{a}\right)$, we have now (instead of (21))

$$
\Lambda_{\mu}^{a}\left(n_{1}, n_{2}, n\right)=\left(\frac{n_{1}}{L_{1}}\right)^{2}+\left(\frac{n_{2}}{L_{2}}\right)^{2}+2 \omega^{a}\left(n+\frac{1}{2}\right)-m_{\mu}^{a}, \quad n_{1}, n_{2} \in \mathbf{Z}, n \in \mathbf{N},
$$

where

$$
m_{t 1}^{a}=m_{t 2}^{a}=0, \quad m_{1}^{a}=-m_{2}^{a}=-2 \omega^{a} .
$$

We can proceed again by writing the corresponding zeta function

$$
\zeta_{\bar{\Theta}}(s)=\frac{\Omega}{2 \pi L_{1} L_{2}} \sum_{a} \omega^{a} \sum_{\mu} \sum_{n} \sum_{n_{1}, n_{2}} \Lambda_{\mu}^{a}\left(n_{1}, n_{2}, n\right)^{-s},
$$

with $\Omega$ the total spacetime volume. Introducing $\bar{L}_{i}^{a} \equiv L_{i} \sqrt{2 \omega^{a}}, i=1,2, \bar{m}_{\mu} \equiv m_{\mu}^{a} /\left(2 \omega^{a}\right)$, we can write

$$
\zeta_{\bar{\Theta}}(s)=\frac{\Omega}{4 \pi L_{1} L_{2}} \sum_{a}\left(2 \omega^{a}\right)^{1-s} \sum_{\mu} \zeta\left(s ; \bar{L}_{i}^{a}, \bar{m}_{\mu}\right),
$$


where

$$
\zeta\left(s ; \bar{L}_{i}^{a}, \bar{m}_{\mu}\right) \equiv \sum_{n=0}^{\infty} \sum_{n_{1}, n_{2}=-\infty}^{+\infty}\left[\left(\frac{n_{1}}{\bar{L}_{1}}\right)^{2}+\left(\frac{n_{2}}{\bar{L}_{2}}\right)^{2}+n+\alpha_{\mu}\right]^{-s},
$$

being $\alpha_{\mu} \equiv 1 / 2-\bar{m}_{\mu}$.

After some work that involves performing, as before, the usual Mellin transform, using later Jacobi's theta function identity on the sums over the indexes $n_{1}$ and $n_{2}$, and integrating back the the Mellin transform (see [7]), we obtain

$$
\begin{aligned}
& -\frac{1}{2} \sum_{\lambda} \zeta\left(s ; L_{i}, \alpha_{\lambda}\right)+\zeta\left(s ; L_{i}, 0\right)=\frac{\Omega}{2 \pi L_{1} L_{2}} \sum_{a, \lambda} \omega^{a}\left(\frac{\mu^{2}}{2 \omega^{a}}\right)^{s}\left[2 \pi L_{1} L_{2} \omega^{a} \zeta\left(s-1 ; \alpha_{\lambda}\right)-\zeta\left(s ; \alpha_{\lambda}\right)\right. \\
& \left.\quad+\frac{2 \pi^{s} L_{1} L_{2}\left(2 \omega^{a}\right)^{(s+1) / 2}}{\Gamma(s)} \sum_{n, n_{1}, n_{2}}\left(\frac{L_{1}^{2} n_{1}^{2}+L_{2}^{2} n_{2}^{2}}{n+\alpha_{\lambda}}\right)^{(s-1) / 2} K_{s-1}\left(2 \pi \sqrt{2 \omega^{a}\left(n+\alpha_{\lambda}\right)\left(L_{1}^{2} n_{1}^{2}+L_{2}^{2} n_{2}^{2}\right)}\right)\right] .
\end{aligned}
$$

Finally, by taking the derivative of this zeta function, for the one-loop effective action density we arrive at the following expression

$$
\begin{aligned}
\frac{\Gamma^{(1)}}{\Omega}\left(L_{1}, L_{2}\right)= & \sum_{a=1}^{N^{2}-1}\left\{\left(\omega^{a}\right)^{2}\left[-\frac{11}{12} \ln \left(\frac{\mu^{2}}{2 \omega^{a}}\right)+\frac{11}{12}-\zeta_{H}^{\prime}\left(-1, \frac{3}{2}\right)-\zeta_{H}^{\prime}\left(-1, \frac{1}{2}\right)+\frac{1}{2} \ln \left(\frac{1}{2}\right)+i \frac{\pi}{2}\right]\right. \\
& +\frac{\omega^{a}}{2 \pi L_{1} L_{2}}(\ln 2-i \pi)+\frac{\sqrt{2}}{\pi}\left(\omega^{a}\right)^{3 / 2} \\
& \left.\cdot \sum_{\mu} \sum_{n, n_{1}, n_{2}} \sqrt{\frac{n+\alpha_{\mu}}{L_{1}^{2} n_{1}^{2}+L_{2}^{2} n_{2}^{2}}} K_{1}\left(2 \pi \sqrt{2 \omega^{a}\left(n+\alpha_{\lambda}\right)\left(L_{1}^{2} n_{1}^{2}+L_{2}^{2} n_{2}^{2}\right)}\right)\right\} .
\end{aligned}
$$

The difference from Eq. (40) is that the present effective action is explicitly given in terms of a quickly (exponentially like) convergent series, as far as $L_{1}, L_{2}>1$. For the first terms of the expansion we get a qualitative coincidence with the large- $\rho$ expansion of the preceding case. This allows us to stop the discussion of the torus case here. In particular, the numerical analysis and that of the imaginary part closely parallel the one carried out in the previous section.

\section{RG-improved gauge field effective potential in an $\mathrm{SU}(2)$ gauge theory in curved spacetime}

Let us consider now the $\mathrm{SU}(2)$ gauge theory with the Lagrangian

$$
\mathcal{L}=-\frac{1}{4} G_{\mu \nu}^{a} G^{\mu \nu a}+a_{1} R^{2}+a_{3} G
$$

on the constant-curvature background $R_{\mu \nu}=\frac{1}{4} R g_{\mu \nu}$, where the curvature is small enough. Such a theory is multiplicatively renormalizable in curved spacetime (for details, see [9]). Here $G$ is the Gauss-Bonnet combination, and $a_{1}, a_{3}$ are the usual gravitational coupling constants.

The covariantly constant gauge field choice may be understood as an expansion in normal coordinates. Then, the first term in this expansion gives the flat space result and the next the curvature corrections: $\frac{1}{4} G_{\mu \nu}^{a} G^{\mu \nu a}=\frac{1}{2} H^{2}+O(R)$. 
Taking into account that the theory under consideration is multiplicatively renormalizable, and solving its renormalization group equations for the effective potential we find (see also [12])

$$
V=\frac{1}{2} \frac{g^{2}}{g^{2}(t)} H^{2}-a_{1}(t) R^{2}-a_{3}(t) G
$$

where $G=\frac{R^{2}}{6}$ and

$$
g^{2}(t)=\frac{g^{2}}{1+\frac{11 g^{2} t}{12 \pi^{2}}}, \quad a_{1}(t)=a_{1}, \quad a_{3}(t)=a_{3}-\frac{62 t}{120(4 \pi)^{2}}
$$

Hence, we have got the RG-improved effective potential making the summation of all the leading logs of perturbation theory. The RG parameter is chosen as the logarithm of the effective gauge mass (compare with (4))

$$
t=\frac{1}{2} \ln \frac{\frac{R}{4}+g H}{\mu^{2}}
$$

Such a choice may also be justified by a direct one-loop calculation near flat-space, and then choosing the standard condition on the vanishing of the logarithmic term in the one-loop effective potential. Note also that from (53) at $R=0$ we get the RG-improved effective potential of paper [1].

Now one can find the minimum of the effective potential, which turns out to be (see also [12)

$$
g H_{\min } \simeq \mu^{2} \exp \left(-\frac{24 \pi^{2}}{11 g^{2}}\right)-\frac{R}{4} .
$$

This vacuum takes into account the gravitational corrections. Moreover, there is a critical value of the curvature determined by $H_{\min }=0$ in (55). Hence, like in third section we see again the possibility of transitions between different phases of the chromomagnetic vacuum. For positive curvatures above the critical value $R_{c}$, the ground state is given by $H_{\min }=0$, while for positive and small negative curvatures below $R_{c}$, the ground state has a nonzero background gauge field $H_{\min } \neq 0$. Note, however, that in the above quasi-local expansion we cannot calculate the imaginary and nonlocal parts of the effective action. Hence, it is not clear whether the above $H_{\text {min }}$ value is the true ground state or just a local minimum as in flat space.

\section{Conclusion}

In the present letter we have discussed the gauge field effective potential for a covariantly constant gauge field in curved spacetime. The calculation has been done in two ways: explicit one-loop evaluation on an $\mathbf{S}^{2} \times \mathbf{R}^{2}$ background using zeta-regularization, and RG-improved formalism using a quasi-local expansion. In both approaches the influence of curvature on the phase structure is clearly seen through the shift of the chromomagnetic vacuum due to curvature corrections (curvature-induced phase transitions). Moreover, numerical estimates on the $\mathbf{S}^{2} \times \mathbf{R}^{2}$ background indicate that for some curvature value the nonzero chromomagnetic vacuum may be stabilized (true ground state). Of course, in order to completely confirm such a gravitational effect on the chromomagnetic vacuum, explicit one-loop calculations on other gravitational backgrounds should be done. Note, however, that for the most interesting spacetimes (like De Sitter space $\mathbf{S}^{4}$ ), where nonzero components of the background field lie in nonflat spacetime directions, 
the spectrum gets extremely complicated and the one-loop effective potential becomes really hard to calculate.

It would also be very interesting to study cosmological applications of the above effective potential, for example, trying to construct an inflationary Universe where $H$ plays the role of scalar inflaton.

\section{Acknowledgements}

We would like to thank J. Ambjørn and R. Percacci for useful discussions. This work has been supported by DGICYT (Spain), project PB93-0035 and grant SAB93-0024, by CIRIT (Generalitat de Catalunya), grant GR94-8001, and by RFBR, project 96-02-16017.

\section{References}

[1] G.K. Savvidy, Phys. Lett. 71B (1977) 133.

[2] N.K. Nielsen and P. Olesen, Nucl. Phys. B144 (1978) 376.

[3] E. Elizalde and J. Soto, Ann. Phys. 162 (1985) 192; Nucl. Phys. B283 (1987) 577; E. Elizalde, Nucl. Phys. B243 (1984) 398;

[4] J. Ambjørn, R.J. Hughes and N.K. Nielsen, Ann. Phys. (NY) 150 (1983) 93; J. Ambjørn and R.J. Hughes, Nucl. Phys. B197 (1982) 113.

[5] J. Ambjørn and P. Olesen, Nucl. Phys. B330 (1990) 193.

[6] E.S. Fradkin, D.M. Gitman and Sh. M. Shvartsman, Quantum Electrodynamics with Unstable Vacuum (Springer-Verlag, Berlin, 1991).

[7] E. Elizalde, S.D. Odintsov, A. Romeo, A.A. Bytsenko and S. Zerbini, Zeta Regularization Techniques with Applications (World Scientific, Singapore, 1994); E. Elizalde, Ten Physical Applications of Spectral Zeta Functions (Springer-Verlag, Berlin, 1995).

[8] I.L. Buchbinder and S.D. Odintsov, Izw. VU Zov, Fiz. (Sov. Phys. Journal) n4 (1983) 46; Yad. Fiz. (Sov. J. Nucl. Phys.) 40 (1984) 1338; Lett. Nuovo Cim. 42 (1985) 379.

[9] I.L. Buchbinder, S.D. Odintsov and I.L. Shapiro, Effective Action in Quantum Gravity, (IOP Publishing, Bristol and Philadelphia, 1992).

[10] A. Salam and J. Strathdee, Nucl. Phys. B90 (1975) 203.

[11] H.P. McKean and I.M. Singer, J. Diff. Geom. 1 (1967) 43.

[12] S.D. Odintsov and R. Percacci, preprint SISSA (1996). 


\section{Figure Caption}

Fig. 1. Re $V$ (solid lines) and $\operatorname{Im} V$ (dashed lines), for several values of $\rho$. ¿From bottom up, the curves correspond to $\rho \rightarrow \infty, \rho=10,3,2.21,2.03,1.5,1$. The value of the gauge coupling is $g=0.1$ and the $\mu^{2}$ scale has been taken $\mu^{2}=g e^{24 \pi^{2} /\left(11 g^{2}\right)}$ so that the minimum of $\operatorname{Re} V$ for $\rho \rightarrow \infty$ takes place at $H=1$. 
E. Elizalde, S.D. Odintsov and A. Romeo, Effective potential for a covariantly constant ...

Fig. 1

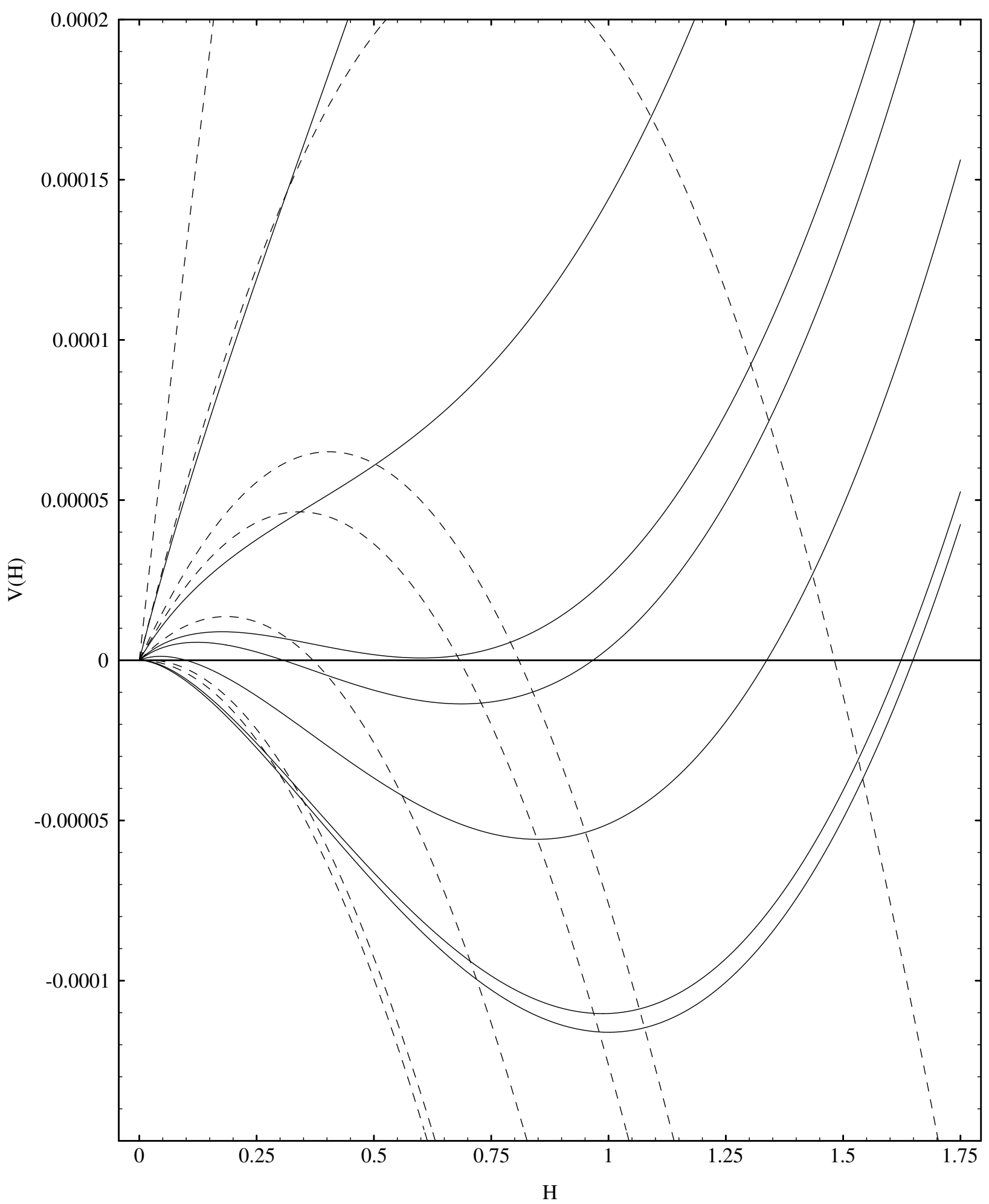

